\title{
Strong Running Coupling from the Gauge Sector of Domain Wall Lattice QCD with Physical Quark Masses
}

\author{
S. Zafeiropoulos, ${ }^{1}$ Ph. Boucaud, ${ }^{2}$ F. De Soto, ${ }^{3,4}$ J. Rodríguez-Quintero, ${ }^{5,4}$ and J. Segovia ${ }^{3,4}$ \\ ${ }^{1}$ Institute for Theoretical Physics, Heidelberg University, Philosophenweg 12, 69120 Heidelberg, Germany \\ ${ }^{2}$ Laboratoire de Physique Théorique (UMR8627), CNRS, Univ. Paris-Sud, Université Paris-Saclay, 91405 Orsay, France \\ ${ }^{3}$ Dpto. Sistemas Físicos, Químicos y Naturales, Univ. Pablo de Olavide, 41013 Sevilla, Spain \\ ${ }^{4}$ CAFPE, Universidad de Granada, E-18071 Granada, Spain \\ ${ }^{5}$ Department of Integrated Sciences and Center for Advanced Studies in Physics, \\ Mathematics and Computation, University of Huelva, E-21071 Huelva, Spain
}

(Received 22 February 2019; published 26 April 2019)

\begin{abstract}
We report on the first computation of the strong running coupling at the physical point (physical pion mass) from the ghost-gluon vertex, computed from lattice simulations with three flavors of domain wall fermions. We find $\alpha_{\overline{\mathrm{MS}}}\left(m_{Z}^{2}\right)=0.1172(11)$, in remarkably good agreement with the world-wide average. Our computational bridge to this value is the Taylor-scheme strong coupling, which has been revealed of great interest by itself because it can be directly related to the quark-gluon interaction kernel in continuum approaches to the QCD bound-state problem.
\end{abstract}

DOI: 10.1103/PhysRevLett.122.162002

Introduction.-Quantum chromodynamics (QCD), the non-Abelian gauge quantum field theory describing the strong interaction between quarks and gluons, can be compactly expressed in one line with a few inputs: namely, the current quark masses and the strong coupling constant, $\alpha_{s}$ [1]. The latter is a running quantity which sets the strength of the strong interaction for all momenta. This running can be, a priori, inferred from the theory and encoded in the renormalization group equation (RGE) of $\alpha_{s}$, the value of which can be thus propagated from one given momentum to any other. The strong coupling is expressed by either the boundary condition for its RGE, generally dubbed $\Lambda_{\mathrm{QCD}}$, or its value at a reference scale, typically the $Z^{0}$-pole mass. This value is considered one of the QCD fundamental parameters, to be fitted from experiments, and amounts to $\alpha_{s}\left(m_{Z}^{2}\right)=0.1181(11)$ [2], in the $\overline{\mathrm{MS}}$ renormalization scheme. Its current uncertainty of about $1 \%$ renders it the least precisely known of all fundamental coupling constants in nature. But at the same time, it is interesting to mention that a plethora of computations of LHC processes depend on an improved knowledge of $\alpha_{s}$ to reduce their theoretical uncertainties [3]. Especially in the Higgs sector, the uncertainty of $\alpha_{s}$ dominates that for the $H \rightarrow c \bar{c}, g g$ branching fractions and, after the error in the bottom mass, the one for the dominant $H \rightarrow b \bar{b}$

Published by the American Physical Society under the terms of the Creative Commons Attribution 4.0 International license. Further distribution of this work must maintain attribution to the author(s) and the published article's title, journal citation, and DOI. Funded by SCOAP ${ }^{3}$. partial decay. And contrarily to other sources of uncertainty, as parton distribution functions, which reduced substantially [4], that for $\alpha_{s}$ has not significantly changed in the last decade. Moreover, the $\alpha_{s}$ running and its uncertainty also has a non-negligible impact in the study of the stability of the electroweak vacuum, in the determination of the unification scale for the interaction couplings and, generally, in discriminating different new physics scenarios.

There are many methods to determine the QCD coupling constant based on precision measurements of different processes and at different energy scales. A description can be found in the last QCD review of the Particle Data Group (PDG) [2] or in specific reviews as, for instance, Ref. [5]. Alternatively, lattice QCD can be applied as a tool to convert a very precise physical observation used for the lattice spacing setting into $\Lambda_{\mathrm{QCD}}$. Thus, lattice QCD calculations can potentially be a great help to increase the accuracy of our knowledge of $\alpha_{s}$. A review of most of the procedures recently implemented to determine the strong coupling from the lattice can be found in Ref. [6]. Among these procedures, there are those based on the computation of QCD Green's functions (see, for instance, Refs. [7-9]), the most advantageous of which exploits the ghost-gluon vertex renormalized in the socalled Taylor scheme [10-17] such that the involved coupling can be computed from two-point Green's functions. As a bonus, this coupling has many phenomenological implications [18-25] and is connected to the quark-gluon interaction kernel in continuum approaches to the QCD bound-state problem [26-30]. In this letter, we shall focus on this method and evaluate the Taylor coupling 
from lattice simulations with three Domain Wall fermions (DWF) at the physical point. DWF (cf. [31,32] for two interesting reviews), owing to their very good chiral properties, are expected to suffer less the impact of discretization artifacts.

The running coupling from QCD two-point Green's functions.-First, we will sketch how the strong running coupling can be obtained from the gauge sector of QCD, invoking only 2-point Green's functions. Let $F$ and $D$ be the form factors (dressing functions) of the ghost and gluon propagators in the Landau gauge; the coupling will then read $[10,13]$

$$
\alpha_{T}\left(k^{2}\right) \equiv \frac{g_{T}^{2}\left(k^{2}\right)}{4 \pi}=\lim _{a \rightarrow 0} \frac{g_{0}^{2}(a)}{4 \pi} F^{2}\left(k^{2}, a\right) D\left(k^{2}, a\right),
$$

renormalized in the Taylor scheme, where $a$ stands for a regularization cutoff (the lattice spacing that is taken to vanish in the end of the calculation). The gauge-field twopoint Green's functions can be obtained from lattice QCD simulations with an extremely high level of accuracy, and combined next as Eq. (1) indicates to produce a precise estimate for the running of the coupling over a large window of momenta. Roughly above 3.5-4 GeV, this running can be very well described by [14]

$$
\begin{aligned}
\alpha_{T}\left(k^{2}\right)= & \alpha_{T}^{\text {pert }}\left(k^{2}\right)\left(1+\frac{9}{k^{2}} R\left(\alpha_{T}^{\text {pert }}\left(k^{2}\right), \alpha_{T}^{\text {pert }}\left(q_{0}^{2}\right)\right)\right. \\
& \left.\times\left(\frac{\alpha_{T}^{\text {pert }}\left(k^{2}\right)}{\alpha_{T}^{\text {pert }}\left(q_{0}^{2}\right)}\right)^{1-\gamma_{0}^{A^{2}} / \beta_{0}} \frac{g_{T}^{2}\left(q_{0}^{2}\right)\left\langle A^{2}\right\rangle_{R, q_{0}^{2}}}{4\left(N_{C}^{2}-1\right)}\right),
\end{aligned}
$$

where the perturbative result is supplemented by a leading operator product expansion (OPE) nonperturbative correction, driven by the dimension-two gluon condensate $g_{T}^{2}\left(q_{0}^{2}\right)\left\langle A^{2}\right\rangle_{R, q_{0}^{2}}$, including its anomalous dimension: $1-\gamma_{0}^{A^{2}} / \beta_{0}=1 / 4$ for $N_{f}=3[33,34]$ and

$$
\begin{aligned}
R\left(\alpha, \alpha_{0}\right)= & \left(1+1.05882 \alpha+1.16814 \alpha^{2}+1.95534 \alpha^{3}\right) \\
& \times\left(1-0.62446 \alpha_{0}-0.26140 \alpha_{0}^{2}-0.04275 \alpha_{0}^{3}\right),
\end{aligned}
$$

obtained here for $N_{f}=3$ as described in the Appendix of Ref. [14]. The momentum $q_{0}=10 \mathrm{GeV}$ is chosen as a subtraction point for the local operator. The perturbative $\alpha_{T}^{\text {pert }}(k)$ can be approximated at the four-loop level by the integration of the $\beta$ function [2], their coefficients being defined in the Taylor scheme $[13,35]$. Thus, the purely perturbative running reads as a function of $\ln \left(k^{2} / \Lambda_{T}^{2}\right)$, where $\Lambda_{T}$ stands for the $\Lambda_{\mathrm{OCD}}$ parameter in the Taylor scheme. The confrontation of lattice results, accurately obtained with Eq. (1), to the running behavior predicted by Eq. (2) allows for a precise determination of the parameters $\Lambda_{T}$ and the gluon condensate $g_{T}^{2}\left(q_{0}^{2}\right)\left\langle A^{2}\right\rangle_{R, q_{0}^{2}}$, both controlling the result displayed by Eq. (2). Finally, as the running coupling in Taylor and $\overline{\mathrm{MS}}$ schemes relate as $\alpha_{T}=\bar{\alpha}\left(1+\left(c_{1} / 4 \pi\right) \bar{\alpha}+\mathcal{O}\left(\bar{\alpha}^{2}\right)\right)$, where $c_{1}$ is known [35], the $\Lambda_{\mathrm{QCD}}$ parameters can be in turn related, owing to their scale independence, by a subtraction of the couplings at asymptotically large momenta, thus obtaining [14]

$$
\frac{\Lambda_{\overline{\mathrm{MS}}}}{\Lambda_{T}}=e^{-\frac{c_{1}}{2 \beta_{0}}}=\exp \left(-\frac{507-40 N_{f}}{792-48 N_{f}}\right) .
$$

All the procedure has been described in detail in a series of articles, resulting from a long-term research program aimed at the determination of $\Lambda_{\overline{\mathrm{MS}}}$ from lattice QCD, where estimates for $N_{f}=0$ [13], $N_{f}=2$ [14], and $N_{f}=2+$ $1+1$ (two degenerate light quarks and two nondegenerate ones with strange and charm flavors) [15-17] have been delivered.

The knowledge of $\Lambda_{\overline{\mathrm{MS}}}$ at a given $N_{f}$ defines the perturbative running of $\alpha_{\overline{\mathrm{MS}}}$, known to give a reliable effective description of the physical world between the energy thresholds of the $N_{f}$ th and $\left(N_{f}+1\right)$ th quark flavors for $N_{f} \geq 3$ [2]. Then, the matching formula

$$
\alpha_{\overline{\mathrm{MS}}}^{N_{f}+1}\left(m_{q}\right)=\alpha_{\overline{\mathrm{MS}}}^{N_{f}}\left(m_{q}\right)\left(1+\sum_{n} c_{n 0}\left(\alpha_{\overline{\mathrm{MS}}}^{N_{f}}\left(m_{q}\right)\right)^{n}\right)
$$

can be applied to extend the running up to the threshold of the $\left(N_{f}+2\right)$ th quark flavor, where $m_{q}$ is the $\overline{\mathrm{MS}}$ running mass of the $\left(N_{f}+1\right)$ th quark and the coefficients $c_{n 0}$ can be found in Refs. [36,37] for $n \leq 4$. One can proceed this way up to the $Z^{0}$ mass scale. Thus, the scale $\Lambda_{T}$, obtained for the running coupling in a Taylor scheme at a given $N_{f}$, can be related to the benchmark value of $\alpha_{\overline{\mathrm{MS}}}\left(m_{Z}^{2}\right)$.

The running coupling at the physical point.-Our previous determinations of $\Lambda_{\overline{\mathrm{MS}}}$ at $N_{f}<3[13,14]$ represented nothing but a heuristic effort, paving the way towards more realistic computations. This is so, first, because the lattice scale setting made by the confrontation with empiric observations is affected by the presence of the physical light flavors, up and down but also strange, thus inducing strong systematic effects. But moreover, even if one estimates and corrects the strange quark deviation in the $N_{f}=2$ case as prescribed in Ref. [27], the matching formula (5) can be hardly trusted at the strange-quark threshold. On the other hand, the one for $N_{f}=2+1+1$ [15-17] cannot be considered as a fully realistic estimate either, as far as it relies on lattice simulations where the lightest pseudoscalar mass ranges from 270 to $510 \mathrm{MeV}$ and where chiral fits were required to take experimental $f_{\pi}$ and $m_{\pi}$ at the physical point $[38,39]$.

We repeat here the analysis with two ensembles of gauge-field configurations with $2+1 \mathrm{DWF}$ simulated at the physical point and a third one with a pion mass of around $300 \mathrm{MeV}$ (see Table I). Furthermore, we follow Ref. [40] and perform a very careful scrutiny of discretization lattice artifacts, which corresponds to taking $a \rightarrow 0$ in 
TABLE I. Setup parameters for the $N_{f}=2+1$ ensembles exploited here [44-48], which are generated with the Iwasaki gauge action [49] and the DWF action [50,51]. The two physical point ensembles use the Möbius kernel [52], while the heavier one uses the Shamir kernel [50,51].

\begin{tabular}{lllll}
\hline \hline$\beta$ & $L^{3} \times T / a^{4}$ & $a^{-1}[\mathrm{GeV}] m_{\pi}[\mathrm{MeV}] m_{\pi} L$ & $\mathrm{~V}\left[\mathrm{fm}^{4}\right]$ & confs \\
\hline
\end{tabular}

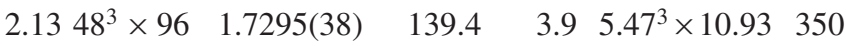

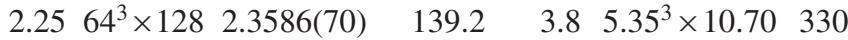

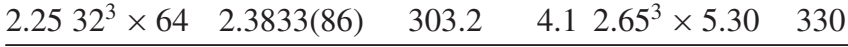

Eq. (1), and approach thus the continuum limit. It can be outlined as follows: bare coupling and dressing functions are combined as Eq. (1) reads and $O(4)$-breaking artifacts cured by applying the $H(4)$-extrapolation [41-43]; residual $O(4)$-invariant artifacts are then removed by identifying $\mathcal{O}\left(a^{2}\right)$ corrections after a thorough comparison of results from the two different simulations at the physical point (as described in Sec. III. B of Ref. [40]); and, finally, the outcome is checked by applying the same $\mathcal{O}\left(a^{2}\right)$ corrections to the third simulation's results.

Figure 1 shows the coupling data for the three ensembles, exhibiting an excellent overlap and displaying a nice running behavior. Besides the good chiral properties of the DWF, a second ace of the exploited ensembles at the physical point is their large physical volume, which is made apparent by the absence of finite-volume effects when their results compare with those for the half-volume third ensemble. The upper bound of the running window, defined by $k a(2.25)=\pi / 2$, corresponds to the largest lattice momenta which, being conservative, can be safely cured for discretization artifacts.

The rightness of Eq. (2) and the need of the gluon condensate $g_{T}^{2}\left(q_{0}^{2}\right)\left\langle A^{2}\right\rangle_{R, q_{0}^{2}}$ for the appropriate description

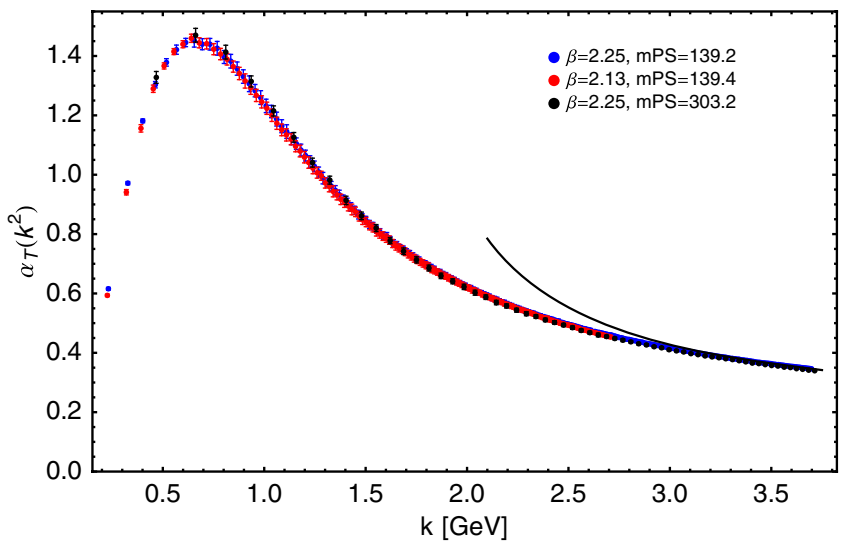

FIG. 1. Running coupling data for the three lattice ensembles of Table I, after being cured for the discretization artifacts. The errors have been obtained by applying the jackknife method. The black solid line displays the result of Eq. (2) for $\Lambda_{T}=581.5 \mathrm{MeV}$, corresponding to $\Lambda_{\overline{\mathrm{MS}}}=320 \mathrm{MeV}$, and $g_{T}^{2}\left(q_{0}^{2}\right)\left\langle A^{2}\right\rangle_{R, q_{0}^{2}}=4.1 \mathrm{GeV}^{2}$. of the momentum running of MOM-renormalized gaugefield Green's functions have been very well established [8,9,13-17,53-56]. Its nature and implications have been also thoroughly investigated in a exhaustive bunch of different analyses [57-70], and its little dependence with the number of dynamical flavors found as well. Indeed, the effect of the heavier flavors can be thought to be negligible. Therefore, we have made the weighted-by-the-errors average of 2.7(1.0) and 4.5(5) $\mathrm{GeV}^{2}$ for the gluon condensate at, respectively, $N_{f}=2$ [14,71] and $N_{f}=4$ [16]; and thus finding for $N_{f}=3, g_{T}^{2}\left(q_{0}^{2}\right)\left\langle A^{2}\right\rangle_{R, q_{0}^{2}}=4.1(1.1) \mathrm{GeV}^{2}$, where the uncertainty has been conservatively estimated by adding the errors in quadrature. We have then applied this value to Eq. (2) and inverted it numerically for all the lattice calculations at the physical point of $\alpha_{T}\left(k^{2}\right)$, with $k>3 \mathrm{GeV}$, and obtained thus the estimates of $\Lambda_{T}$, converted to $\Lambda_{\overline{\mathrm{MS}}}$ through Eq. (4) and displayed in Fig. 2. The plot shows a slow systematic decreasing below $3.62 \mathrm{GeV}$ which, as proven in Refs. [16,17] for $N_{f}=4$, reflects that in this range higher-order nonperturbative corrections need to be included in Eq. (2). Above $3.62 \mathrm{GeV}$, a small plateau appears: 11 points for which their central values differ as much as one per mil, their statistical errors being of the order of $1 \%$. However, the plateau is too small to apply the same fitting strategy developed in Refs. [16,17], with two free parameters and the lowest bound of the fitting window to be determined by the minimization of $\chi^{2}$. We make here no fit but take from literature the value of the condensate and evaluate $\Lambda_{\overline{\mathrm{MS}}}$ instead from the largest available momentum (We have performed correlated and uncorrelated fits for $\Lambda_{\overline{\mathrm{MS}}}$ within this small plateau, extracting central values with similar statistical errors and differing less than $2 \mathrm{MeV}$; and similarly for the derivative of $\Lambda_{\overline{\mathrm{MS}}}$, with the central value appearing to be of the order of $5 \mathrm{MeV} / \mathrm{GeV}$ and the result compatible with zero. All strongly corroborating

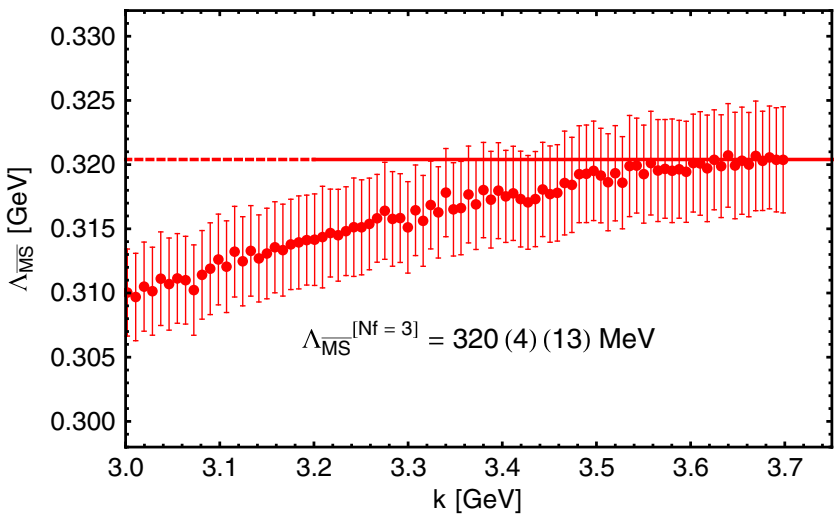

FIG. 2. Estimates of $\Lambda_{\overline{\mathrm{MS}}}$ (red solid circles) for all the lattice calculations of $\alpha_{T}\left(k^{2}\right)$ with $k>3 \mathrm{GeV}$, made through the numerical inversion of Eq. (2) with $g_{T}^{2}\left(q_{0}^{2}\right)\left\langle A^{2}\right\rangle_{R, q_{0}^{2}}=4.1(1.1)$. The error bars displayed in the plot correspond to the propagation of the uncertainty in the lattice determination of $\alpha_{T}$. 
that a plateau is reached. We prefer however to make no fit, take the largest momenta for the estimate and, being conservative, propagate the large uncertainty for the gluon condensate to our final error budget and estimate the possible impact of higher-order nonperturbative corrections.): $\Lambda_{\overline{\mathrm{MS}}}=320(4)(13) \mathrm{MeV}$; the first quoted error results from propagating the one of $\alpha_{T}$ for this momentum into $\Lambda_{\overline{\mathrm{MS}}}$ determined by Eq. (2), while the second one propagates the larger uncertainty from the condensate value. The central values for $\Lambda_{\overline{\mathrm{MS}}}$ and the condensate applied to Eq. (2) produce the black solid curve in Fig. 1.

Beyond this, one can also incorporate the same sort of higher-order nonperturbative correction effectively identified in Ref. [16] and try thus the same fit made therein. In so doing, one would obtain a nice plateau for momenta ranging from 2 to $3.7 \mathrm{GeV}$ and a very consistent best fit for $\Lambda_{\overline{\mathrm{MS}}}=313 \mathrm{MeV}$, which will be used here only to estimate an uncertainty of $7 \mathrm{MeV}$ resulting from the possible impact of higher-order nonperturbative corrections. Thus, following the matching procedure described in the previous section, we will be left with

$$
\alpha_{\overline{\mathrm{MS}}}\left(m_{Z}^{2}\right)=0.1172(3)(9)(5),
$$

where the first error propagates the uncertainty in the lattice determination of the Taylor coupling, the second does so for the value of the condensate and the last one stands for higher-order nonperturbative corrections.

$N_{f}=2+1+1$ versus $N_{f}=2+1$ results. - Let us complete this analysis by relating the current results with our previous ones for $N_{f}=2+1+1$ [16]. The lattice actions employed for the fermionic sector differ, twisted mass for the latter and DWF for the former, although consistent results from both are expected in the continuum limit, if all discretization artifacts are indeed under control. The benchmark value of $\alpha_{\overline{\mathrm{MS}}}\left(m_{\mathrm{Z}}^{2}\right)$ here is $0.1172(11)$, all the errors combined in quadrature, and 0.1200(14) in Ref. [16]; both compatible with the current PDG world average [2], 0.1181(11), but not with each other within their $1-\sigma$ errors. This little difference might be due to a simple statistical deviation but can also reflect a small systematic effect in Ref. [16], caused by the larger pion mass (The pion mass effect on the UV momentum running is seen to be very small in Fig. 1, but effects on the physical scale setting and on the impact of the discretization artifacts cannot be excluded. Specially the latter would require a very accurate control of the continuum limit that might not have been achieved in Ref. [16].). This new updated result from the Taylor coupling, now at the physical point, lies closer to the FLAG lattice average (It includes determinations from current two-point correlators [72-74], Schrödinger functional [75], and Wilson loops [76]): 0.11823(81) [6,77]; but even closer to the nonlattice average of PDG: $0.1174(16)$ [2]. The PDG lattice unweighted average is in turn 0.1188(13), including the ghost-gluon determination
$[16,17]$ among a few others [72-76]. However, updating for the ghost gluon with the current result, one is left with $0.1184(13)$, closer to the FLAG central value. A very accurate $\alpha_{\overline{\mathrm{MS}}}\left(m_{Z}^{2}\right)=0.11852(84)$, obtained from the Schödinger functional and renormalized couplings defined via the gradient flow, has been also recently reported [78], with which our estimate agrees as well. It is noteworthy that this agreement demonstrates and strongly confirms the approaches being radically different, that lattice systematics are well under control. In Ref. [79] DWF have also been employed for the extraction of $\alpha_{s}$ from the hadronic vacuum polarization function albeit not at the physical point. Their result is less precise, 0.1181(27), but anyhow in good agreement with ours.

Beyond this, Fig. 3 displays a direct and striking comparison of the Taylor couplings for $N_{f}=2+1$ and $2+1+1$. It is very apparent that, for momenta above the charm quark mass threshold, the three-flavors coupling decreases faster than the four-flavors one, extending down to nonperturbative momenta a well-known perturbative result: the beta function, the logarithmic derivative of the coupling with opposite sign, lessens when the number of flavors gets bigger. Around the charm threshold and below, within the deep IR domain, four- and three-flavors couplings appear to be the same, both reaching strikingly the same peak. Lightened by a few recent works [26-30], bridging the gauge sector and phenomenological applications in QCD in connection with the bound-state problem, this feature can be well understood: the Taylor coupling can be related to the quark-gluon interaction kernel [27], both differing only by a small correction rooting in the ghost sector and not depending very much on the number of flavors. Figure 3 thereby implies that the IR quark-gluon interaction strength does not depend on whether the charm quark becomes active or not.

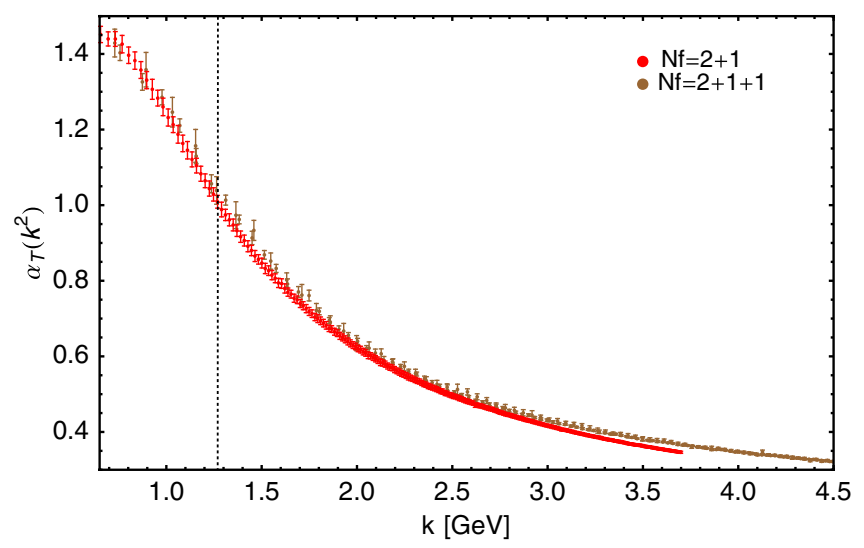

FIG. 3. Taylor coupling from the lattice, in the continuum limit, for $N_{f}=2+1 \mathrm{DW}$ dynamical flavors (red solid circles) and for $N_{f}=2+1+1$ twisted-mass flavors (brown solid circles) taken from Ref. [16]. The dotted line indicates the charm quark threshold at its $\overline{\mathrm{MS}}$ running mass. 
Although expected, to our knowledge, this outcome has never been so remarkably exposed.

Conclusions. - In this Letter we presented our results on the first computation of the strong running coupling at the physical point from the ghost-gluon vertex, computed from lattice simulations with $N_{f}=2+1$ DWF. We therewith update the last results $[16,17]$ obtained from applying the same procedure with four flavors but relatively large pion mass. The continuum limit treatment has been also herein improved. Thus, we have been left with an estimate for the benchmark value of $\alpha_{\overline{\mathrm{MS}}}\left(m_{Z}^{2}\right)$ more accurate, closer to the central value of the current lattice (FLAG) average [6], and in remarkably good agreement with the nonlattice average of the PDG [2]. Moreover, lattice and nonlattice averages of the PDG would come closer after updating the ghost-gluon determination. The convergence of lattice and nonlattice averages is very welcome, implying first that theory meets experiments but, not less important, that systematics effects from the discretization are under control and one can thereby take at face value the lattice errors, approaching thus the goal of getting the $\alpha_{\overline{\mathrm{MS}}}\left(m_{Z}^{2}\right)$ uncertainty below the $1 \%$ level.

On the other hand, the strong coupling in the Taylor scheme, by itself, is an interesting quantity, as it can be directly related to the quark-gluon interaction kernel in continuum approaches to the QCD bound-state problem. It has been herein obtained for three dynamical quarks at the physical point, and has been shown to compare very well with previous results for four dynamical quarks but nonphysical pion mass, qualitatively but also quantitatively, beyond the small deviations due, presumably, to the larger pion mass, which impacts on the very delicate extraction of $\alpha_{\overline{\mathrm{MS}}}\left(m_{Z}^{2}\right)$. Such a comparison shows that the activation of the charm quark does not significantly affect the infrared quark-gluon interaction strength, and it only makes the running coupling decrease slower, above the charm threshold, as suggested by the $\beta$ function.

We are indebted to the RBC/UKQCD Collaboration, especially to Peter Boyle, Norman Christ, Zhihua Dong, Chulwoo Jung, Nicolas Garron, Bob Mawhinney, and Oliver Witzel, for access to the lattices used in this work and to Rainer Sommer for fruitful discussions. We thank the support of the MINECO FPA2017-86380-P Grant, and that of the DFG Collaborative Research Centre SFB 1225 (ISOQUANT) (S.Z.). Numerical computations used resources of CINES, GENCI IDRIS (Project id 52271), and of the IN2P3 computing facility in France.

[1] F. Wilczek, Phys. Today 53, No. 8, 22 (2000).

[2] M. Tanabashi et al. (Particle Data Group), Phys. Rev. D 98, 030001 (2018).

[3] C. Anastasiou, C. Duhr, F. Dulat, F. Herzog, and B. Mistlberger, Phys. Rev. Lett. 114, 212001 (2015).
[4] R. D. Ball, S. Carrazza, L. Del Debbio, S. Forte, Z. Kassabov, J. Rojo, E. Slade, and M. Ubiali (NNPDF Collaboration), Eur. Phys. J. C 78, 408 (2018).

[5] S. Bethke, A. H. Hoang, S. Kluth, J. Schieck, I. W. Stewart et al., arXiv:1110.0016.

[6] S. Aoki et al., Eur. Phys. J. C 77, 112 (2017).

[7] B. Allés, D. Henty, H. Panagopoulos, C. Parrinello, C. Pittori, and D. G. Richards, Nucl. Phys. B502, 325 (1997).

[8] P. Boucaud et al., J. High Energy Phys. 04 (2000) 006.

[9] P. Boucaud, J. P. Leroy, J. Micheli, O. Pène, H. Moutarde, C. Roiesnel, and J. Rodríguez-Quintero, J. High Energy Phys. 01 (2002) 046.

[10] A. Sternbeck, K. Maltman, L. von Smekal, A. G. Williams, E. M. Ilgenfritz, and M. Muller-Preussker, Proc. Sci., LATTICE2007 (2007) 256.

[11] A. Sternbeck et al., Proc. Sci., LAT2009 (2009) 210.

[12] A. Sternbeck, K. Maltman, M. Muller-Preussker, and L. von Smekal, Proc. Sci., LATTICE2012 (2012) 243.

[13] P. Boucaud, F. De Soto, J. P. Leroy, A. Le Yaouanc, J. Micheli, O. Pène, and J. Rodríguez-Quintero, Phys. Rev. D 79, 014508 (2009).

[14] B. Blossier, P. Boucaud, F. De soto, V. Morenas, M. Gravina, O. Pène, and J. Rodríguez-Quintero (ETM Collaboration), Phys. Rev. D 82, 034510 (2010).

[15] B. Blossier, P. Boucaud, M. Brinet, F. De Soto, X. Du, M. Gravina, V. Morenas, O. Pene, K. Petrov, and J. RodriguezQuintero, Phys. Rev. D 85, 034503 (2012).

[16] B. Blossier, P. Boucaud, M. Brinet, F. De Soto, X. Du, V. Morenas, O. Pene, K. Petrov, and J. Rodriguez-Quintero, Phys. Rev. Lett. 108, 262002 (2012).

[17] B. Blossier, P. Boucaud, M. Brinet, F. De Soto, V. Morenas, O. Pene, K. Petrov, and J. Rodriguez-Quintero, Phys. Rev. D 89, 014507 (2014).

[18] A. C. Aguilar, D. Binosi, J. Papavassiliou, and J. RodriguezQuintero, Phys. Rev. D 80, 085018 (2009).

[19] A. Aguilar, D. Binosi, and J. Papavassiliou, J. High Energy Phys. 07 (2010) 002.

[20] A. C. Aguilar, D. Ibanez, and J. Papavassiliou, Phys. Rev. D 87, 114020 (2013).

[21] A. C. Aguilar, M. N. Ferreira, C. T. Figueiredo, and J. Papavassiliou, Phys. Rev. D 99, 034026 (2019).

[22] A. C. Aguilar and C. T. Figueiredo, in Proceedings of the 14th International Workshop on Hadron Physics (Hadron Physics 2018) Florianopolis, Santa Catarina, Brazil, 2018 (2018).

[23] M. Q. Huber and L. von Smekal, J. High Energy Phys. 04 (2013) 149.

[24] M. Q. Huber, Eur. Phys. J. C 77, 733 (2017).

[25] M. Q. Huber, arXiv:1808.05227.

[26] D. Binosi, L. Chang, J. Papavassiliou, and C. D. Roberts, Phys. Lett. B 742, 183 (2015).

[27] D. Binosi, C. D. Roberts, and J. Rodriguez-Quintero, Phys. Rev. D 95, 114009 (2017).

[28] D. Binosi, C. Mezrag, J. Papavassiliou, C. D. Roberts, and J. Rodriguez-Quintero, Phys. Rev. D 96, 054026 (2017).

[29] J. Rodriguez-Quintero, D. Binosi, C. Mezrag, J. Papavassiliou, and C. D. Roberts, Few-Body Syst. 59, 121 (2018). 
[30] D. Binosi, L. Chang, M. Ding, F. Gao, J. Papavassiliou, and C. D. Roberts, Phys. Lett. B 790, 257 (2019).

[31] P. M. Vranas, Nucl. Phys. B, Proc. Suppl. 94, 177 (2001).

[32] D. B. Kaplan, in Modern perspectives in lattice QCD: Quantum field theory and high performance computing, Proceedings of the International School, 93rd Session, Les Houches, France, 2009 (2009), pp. 223-272.

[33] J. A. Gracey, Phys. Lett. B 552, 101 (2003).

[34] K. Chetyrkin and A. Maier, J. High Energy Phys. 01 (2010) 092.

[35] K. Chetyrkin and A. Retey, arXiv:hep-ph/0007088.

[36] K. Chetyrkin, J. H. Kuhn, and C. Sturm, Nucl. Phys. B744, 121 (2006).

[37] B. A. Kniehl, A. V. Kotikov, A. I. Onishchenko, and O. L. Veretin, Phys. Rev. Lett. 97, 042001 (2006).

[38] R. Baron, P. Boucaud, J. Carbonell, A. Deuzeman, V. Drach et al. (ETM Collaboration), J. High Energy Phys. 06 (2010) 111.

[39] R. Baron, B. Blossier, P. Boucaud, J. Carbonell, A. Deuzeman et al. (ETM Collaboration), Proc. Sci., LATTICE2010 (2010) 123.

[40] P. Boucaud, F. De Soto, K. Raya, J. Rodriguez-Quintero, and S. Zafeiropoulos, Phys. Rev. D 98, 114515 (2018).

[41] D. Becirevic, P. Boucaud, J. P. Leroy, J. Micheli, O. Pene, J. Rodriguez-Quintero, and C. Roiesnel, Phys. Rev. D 60, 094509 (1999).

[42] D. Becirevic, P. Boucaud, J. P. Leroy, J. Micheli, O. Pene, J. Rodriguez-Quintero, and C. Roiesnel, Phys. Rev. D 61, 114508 (2000).

[43] F. de Soto and C. Roiesnel, J. High Energy Phys. 09 (2007) 007.

[44] C. Allton et al. (RBC and UKQCD Collaborations), Phys. Rev. D 76, 014504 (2007).

[45] C. Allton et al. (RBC and UKQCD Collaborations), Phys. Rev. D 78, 114509 (2008).

[46] R. Arthur et al. (RBC and UKQCD Collaborations), Phys. Rev. D 87, 094514 (2013).

[47] T. Blum et al. (RBC and UKQCD Collaborations), Phys. Rev. D 93, 074505 (2016).

[48] P. A. Boyle, L. Del Debbio, A. Jttner, A. Khamseh, F. Sanfilippo, and J. T. Tsang, J. High Energy Phys. 12 (2017) 008 .

[49] Y. Iwasaki, Nucl. Phys. B258, 141 (1985).

[50] D. B. Kaplan, Phys. Lett. B 288, 342 (1992).

[51] Y. Shamir, Nucl. Phys. B406, 90 (1993).

[52] R. C. Brower, H. Neff, and K. Orginos, Nucl. Phys. B, Proc. Suppl. 140, 686 (2005).

[53] P. Boucaud, A. Le Yaouanc, J. Leroy, J. Micheli, O. Pène, and J. Rodríguez-Quintero, Phys. Lett. B 493, 315 (2000).

[54] P. Boucaud, A. Le Yaouanc, J. P. Leroy, J. Micheli, O. Pène, and J. Rodríguez-Quintero, Phys. Rev. D 63, 114003 (2001).

[55] P. Boucaud, J. Leroy, A. Le Yaouanc, A. Lokhov, J. Micheli, O. Pène, J. Rodríguez-Quintero, and C. Roiesnel, J. High Energy Phys. 01 (2006) 037.
[56] P. Boucaud, M. Brinet, F. De Soto, V. Morenas, O. Pène, K. Petrov, and J. Rodriguez-Quintero, J. High Energy Phys. 04 (2014) 086.

[57] F. V. Gubarev and V. I. Zakharov, Phys. Lett. B 501, 28 (2001).

[58] P. Boucaud, J. P. Leroy, A. Le Yaouanc, J. Micheli, O. Pène, F. De Soto, A. Donini, H. Moutarde, and J. RodríguezQuintero, Phys. Rev. D 66, 034504 (2002).

[59] E. Ruiz Arriola, P. O. Bowman, and W. Broniowski, Phys. Rev. D 70, 097505 (2004).

[60] D. Dudal, R. F. Sobreiro, S. P. Sorella, and H. Verschelde, Phys. Rev. D 72, 014016 (2005).

[61] E. Megias, E. Ruiz Arriola, and L. L. Salcedo, J. High Energy Phys. 01 (2006) 073.

[62] P. Boucaud, F. de Soto, J. P. Leroy, A. Le Yaouanc, J. Micheli, H. Moutarde, O. Pène, and J. Rodríguez-Quintero, Phys. Rev. D 74, 034505 (2006).

[63] E. Ruiz Arriola and W. Broniowski, Phys. Rev. D 73, 097502 (2006).

[64] K.-I. Kondo, Phys. Rev. D 74, 125003 (2006).

[65] E. Megias, E. Ruiz Arriola, and L. L. Salcedo, Phys. Rev. D 75, 105019 (2007).

[66] E. Megias, E. Ruiz Arriola, and L. L. Salcedo, Phys. Rev. D 80, 056005 (2009).

[67] D. Dudal, O. Oliveira, and N. Vandersickel, Phys. Rev. D 81, 074505 (2010).

[68] D. Dudal, S. Sorella, and N. Vandersickel, Phys. Rev. D 84, 065039 (2011).

[69] L. Chang, C. D. Roberts, and P. C. Tandy, Phys. Rev. C 85, 012201(R) (2012).

[70] P. Boucaud, D. Dudal, J. P. Leroy, O. Pene, and J. Rodriguez-Quintero, J. High Energy Phys. 12 (2011) 018.

[71] We have also considered the $6 \%$ of correction resulting from the strange quark in the lattice scale setting prescribed by Ref. [27].

[72] A. Bazavov, N. Brambilla, X. Garciai Tormo, P. Petreczky, J. Soto, and A. Vairo, Phys. Rev. D 90, 074038 (2014).

[73] B. Chakraborty, C. T. H. Davies, B. Galloway, P. Knecht, J. Koponen, G. C. Donald, R. J. Dowdall, G. P. Lepage, and C. McNeile, Phys. Rev. D 91, 054508 (2015).

[74] C. McNeile, C. T. H. Davies, E. Follana, K. Hornbostel, and G. P. Lepage, Phys. Rev. D 82, 034512 (2010).

[75] S. Aoki et al. (PACS-CS Collaboration), J. High Energy Phys. 10 (2009) 053.

[76] K. Maltman, D. Leinweber, P. Moran, and A. Sternbeck, Phys. Rev. D 78, 114504 (2008).

[77] S. Aoki et al. (Flavour Lattice Averaging Group), arXiv: 1902.08191.

[78] M. Bruno, M. Dalla Brida, P. Fritzsch, T. Korzec, A. Ramos, S. Schaefer, H. Simma, S. Sint, and R. Sommer (ALPHA Collaboration), Phys. Rev. Lett. 119, 102001 (2017).

[79] R. J. Hudspith, R. Lewis, K. Maltman, and E. Shintani, arXiv:1804.10286. 